\title{
On the Oscillations of Statically Indeterminate Beams
}

\author{
By \\ B. II. Karnopp, Ann Arbor, Mich., and B. Nagendra, Toronto, Canada \\ With 2 Figures
}

(Received December 2, 1968)

Summary - Zusammenfassung

On the Oscillations of Statically Indeterminate Beams. The problem of determining the natural frequencies and modes of a statically indeterminant, Timoshenko beam is considered. By lumping the beam properties of linear and rotary inertia at discrete points along the length of the beam and by employing the complementary, variational principle, an approximate solution is obtained by simple matrix iteration.

Über die Schwingungen statisch unbestimmter Balken. Das Problem der Bestimmung von Eigenfrequenzen und Schwingungsformen eines statisch unbestimmten Trmoshenkoträgers wird behandelt. Durch Zusammenziehung der Trägereigenschaften von Masse und. Drehmasse an diskreten Punkten entlang des Trägers und durch Anwendung des komplementären Variationsprinzips wird eine Näherungslösung durch einfache Matrizeniteration erreicht.

\section{Introduction}

In a recent paper [1], the problem of determining the natural frequencies and shear and moment modes of a Trmoshenko beam of variable cross section was considered. The properties of linear and rotary inertia were considered to be lumped at discrete points along the beam. By an application of the complementary variational principle, the problem of determining natural frequencies was reduced to a simple matrix iteration. In addition, straightforward bounds on the computation were derived.

In the present paper, we generalize the method of [1] to cover the case of statically indeterminant, variable cross section, Trmoshenko beams. We will also consider problems in which the beam is required to carry point masses or rotary inertia elements. We consider only the case in which these elements occur at the end of a beam section, but the generalization to cases in which these elements occur along the length of the beam will be obvious.

\section{Formulation of the Problem}

We begin by considering a general beam element; see Fig. 1. As in [1] we consider sections of equal mass and equal rotary inertia, defining 


$$
x_{i}{ }^{\prime}: \int_{0}^{x_{i}{ }^{\prime}}(\gamma A / g) d x=\mathrm{im}, \quad y_{i}^{\prime}: \int_{0}^{y_{i}{ }^{\prime}}(\gamma I / g) d x=i \eta
$$

where

$$
m=\left[\int_{0}^{L}(\gamma A / g) d x / n\right], \quad \eta=\left[\int_{0}^{L}(\gamma I / g) d x / n\right] .
$$

Here $(\gamma / g)$ is the mass density, $A$ the cross sectional aroa, and $I$ the moment of inertia of the cross section.

As in [1] we consider point masses, $m$, to be placed at positions, $x_{i}$, and point, rotary inertia elements, $\eta$, to be placed at positions, $y_{i}$. Here we also choose $x_{i}$ to be the center of mass of the section from $x_{i-1}^{\prime}$ to $x_{i}{ }^{\prime}$, and $y_{i}$ is analogously taken to be the center of inertia of the section from $y_{i}{ }^{\prime}-1$ to $y_{i}{ }^{\prime}$ :

$$
x_{i}=\int_{x_{i}^{\prime}-1}^{x_{i}{ }^{\prime}}(\gamma A / g) x d x / m ; \quad y_{i}=\int_{y_{i}^{\prime}-1}^{y_{i}^{\prime}}(\gamma I / g) x d x / \eta .
$$

We now construct a free body diagram for the system; Fig. 1. At the point $x_{i}$, we label the force between $m$ and the beam by $\dot{r}_{i}$, and at

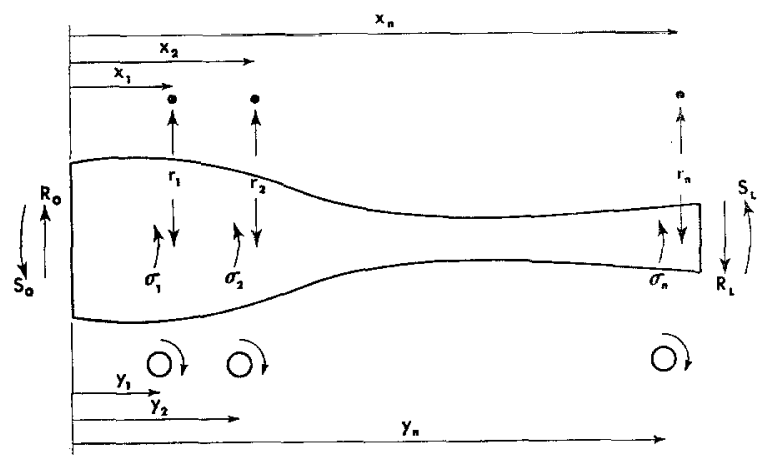

Fig. 1. Free Body Diagram

the point $y_{i}$ we label the torque reaction between $\eta$ and the beam by $\dot{\sigma}_{i}$. We then rename the $y_{i}$

$$
y_{i}=x_{i+n}
$$

and scale and redefine the $\sigma_{i}$

$$
\sigma_{i}=(\eta / m)^{\mathbf{1} / \mathbf{2}} r_{i+n}
$$

so that the kinetic energy $T$

$$
T=(1 / 2 m) \sum_{i=1}^{n} r_{i}^{2}+(1 / 2 \eta) \sum_{i=1}^{n} \sigma_{i}^{2}
$$


becomes

$$
T=(1 / 2 m) \sum_{i=1}^{2 n} r_{i}^{2}
$$

The potential coenergy, $V^{*}$, can then be written as

$$
V^{*}=\frac{1}{2} \int_{0}^{L}\left[\left(M^{2} / E I\right)+\left(Q^{2} / k A G\right)\right] d x,
$$

where $M$ and $Q$ are the moment and shear distributions in the beam:

$$
\begin{aligned}
M(x) & =\sum_{i=1}^{n} \dot{\sigma}_{i} U\left(y_{i}-x\right)-\sum_{i=1}^{n} \dot{r}_{i}\left(x_{i}-x\right) U\left(x_{i}-x\right)- \\
& \quad-\dot{R}_{L}(L-x)+\dot{S}_{L} \\
& =-\sum_{i=1}^{n} \dot{r}_{i}\left(x_{i}-x\right) U\left(x_{i}-x\right)+(\eta / m)^{1 / 2} \sum_{i=n+1}^{2 n} \dot{r}_{i} U\left(x_{i}-x\right)- \\
Q(x) & =\sum_{i=1}^{n} \dot{r}_{i} U\left(x_{i}-x\right)+\dot{R}_{L},
\end{aligned}
$$

where $U$ is the Heaviside function

$$
\begin{aligned}
U(x) & =0 & & x<0, \\
& =1 & & x \geqslant 0 .
\end{aligned}
$$

Thus inserting (7) and (8) into (6) yields

$$
V^{*}=V^{*}\left(\dot{r}_{1}, \dot{r}_{2}, \ldots, \dot{r}_{2 n}, \dot{R}_{L}, \dot{S}_{L}\right)
$$

a quadratic form.

Not all the variables in (9) are independent. The conditions of equilibrium and any geometric constraints must be met before we employ (9) to obtain system equations. The procedure we follow for various edge conditions is indicated below:

(a) $x=0$, Built In: Here $R_{0} \neq 0, S_{0} \neq 0$. We can assume that these have been climated by means of equilibrium. If either $R_{L}$ or $S_{L}$ are nonzero, these can then be eliminated through Castiglano's principle.

(b) $x=0$, Pinned: Here $R_{0} \neq 0, S_{0}=0$. Assume $R_{0}$ has been eliminated by equilibrium. We then eliminated either $R_{L}$ or $S_{L}$ by another equilibrium condition, presumably a moment sum about $x=0$ in order that $R_{0}$ will not appear. If both $R_{L}$ and $S_{L}$ are non-zero, we eliminate the remaining variable through Castigliano's theorem.

(c) $x=0$, Sliding: Here $R_{0}=0, S_{0} \neq 0$. Assume $S_{0}$ has been eliminated through one equilibrium condition and eliminate either $R_{L}$ or $S_{L}$ 
through the other equilibrium condition, presumably the sum of the forces equals zero. If, however, $R_{L}=0$, both equilibrium conditions will have to actually be employed since one will need to use a moment condition to determine $S_{L}$ and this will involve $S_{0}$.

Once the equilibrium conditions have been met, any remaining reactions can be determined through Castigutano's principle.

(d) $x=0$, Free: If $x=L$ is a free end, all end reactions are zero, and, for example, $r_{1}$ and $\sigma_{1}$ can be eliminated through equilibrium. If $x=L$ is either pinned or sliding, only one interior variable need be eliminated to satisfy equilibrium. If $x=L$ is built in, we have case (a).

Orce $R_{L}$ and $S_{L}$ have been eliminated we obtain $V^{*}$ from (9) as a quadratic form :

$$
V^{*}=\frac{1}{2} \sum_{i=1}^{2 n} \sum_{j=1}^{2 n} A_{i j} \dot{r}_{i} \dot{r}_{j} .
$$

By (5) and (10) and the complementary principle

$$
\delta \int_{t_{q}}^{t_{1}}\left(T-V^{*}\right) d t=0 \quad \delta r_{i}=0 \text { at } t_{0}, t_{1}
$$

we obtain the following system equations:

$$
\sum_{j=1}^{2 n} A_{i j} \ddot{r}_{j}+(1 / m) r_{i}=0
$$

and letting $r_{i}=R_{i} \sin p t$ we get

$$
\sum_{j=1}^{2 n} A_{i j} R_{j}=\left(1 / m p^{2}\right) R_{i} .
$$

The equations (11) are the same form as those obtained in [1]. Thus we may perform an iteration which will converge on the lowest natural frequency and then use a sweeping technique in conjunction with iteration to obtain the higher frequencies. Lower bounds may again be obtained.

Finally as a practical note, we can say from experience that if we wish to determine $m$ frequencies, we need to use $n=2 \mathrm{~m}$ subdivisions as a minimum. That means the matrix $[A]$ will be $4 \mathrm{~m} \times 4 \mathrm{~m}$.

\section{Examples}

To illustrate the above procedure and the degree of accuracy which can be obtained, we consider several examples.

Example 1: Built-in-Pinned Timoshwnko Beam. We consider a uniform beam defined by the following parameters:

$$
\begin{aligned}
& b^{2}=\left(\gamma A L^{4} p^{2} / E I g\right) \\
& r^{2}=\left(I / A L^{2}\right) \\
& s^{2}=\left(E I / k A G L^{2}\right) .
\end{aligned}
$$


For this case we have

$$
m=(\gamma A L / n g), \quad \eta=(\gamma I L / n g),
$$

and the positions $x_{i}=y_{i}=x_{i+n}$.

We form $M(x)$ and $Q(x)$ as in (7) and (8) noting that we now have $S_{L}=0$. We assume the $R_{0}$ and $S_{0}$ have been eliminated through the equilibrium conditions. $R_{L}$ remains to be eliminated through Castialiano's principle. The condition is

$$
\frac{\partial V^{*}}{\partial \dot{R}_{L}}=0 .
$$

Noting that in terms of (a) we have

$$
V^{*}=(1 / 2 E I) \int_{0}^{L}\left[M^{2}+s^{2} L^{2} Q^{2}\right] d x,
$$

the condition (b) becomes

$$
\begin{aligned}
\dot{R}_{L}=\sum_{i=1}^{n} \dot{r}_{i}[ & \left.-s^{2}\left(x_{i} / L\right)-\frac{1}{2}\left(x_{i} / L\right)^{2}+\frac{1}{6}\left(x_{i} / L\right)^{3}\right] /\left(s^{2}+\frac{1}{3}\right)+ \\
& +r \sum_{i=n+1}^{2 n} \dot{r}_{i}\left[\left(x_{i} / L\right)-\frac{1}{2}\left(x_{i} / L\right)^{2}\right] /\left(s^{2}+\frac{1}{3}\right) .
\end{aligned}
$$

Inserting (c) into $V^{*}$, we obtain $[A]$ as

$$
\begin{gathered}
A_{i j}=(1 / E I)\left\{x_{k}\left[s^{2} L^{2}+x_{i} x_{j}-\frac{1}{2}\left(x_{i}+x_{j}\right) x_{k}+\frac{1}{3} x_{k}{ }^{2}\right]-\left(L^{3} / f\right) w_{i} w_{j}\right\} \\
0<i \leqslant n \quad n<j \leqslant 2 n \\
A_{i j}=A_{j i}=(1 / E I) r L\left\{x_{k}\left(x_{i}-\frac{1}{2} x_{k}\right)-L^{2} w_{i} w_{j} / f\right\} \\
n<i \leqslant 2 n \quad n<j \leqslant 2 n \\
A_{i j}=(1 / E I)(r L)^{2}\left[x_{k}-L u_{i} u_{j} / f\right]
\end{gathered}
$$

where

$$
\begin{aligned}
f & =\left(s^{2}+\frac{1}{3}\right) \\
u_{i} & =\left(x_{i} / L\right)-\frac{1}{2}\left(x_{i} / L\right)^{2} \\
w_{i} & =-s^{2}\left(x_{i} / L\right)-\frac{1}{2}\left(x_{i} / L\right)^{2}+\frac{1}{6}\left(x_{i} / L\right)^{3} . \\
x_{k} & =\min \left(x_{i}, x_{j}\right) .
\end{aligned}
$$

The particular case of $r=0.02, s=0.05$ for $n=10$ and $n=20$ subdivisions was considered, and the first five values of (b) were compared to the exact values of Huang [2]. 


$\begin{array}{cccr}\text { Mode } & r=0.02 & s=0.05 & \\ & n=10 & n=20 & \text { Exact }(\mathrm{b}) \\ 1 & 14.88392448 & 14.88667536 & \\ 2 & 45.63749790 & 45.71701574 & 44.88754392 \\ 3 & 88.39642429 & 88.98894596 & 89.74295800 \\ 4 & 139.24242020 & 140.63797569 & 141.36942542 \\ 5 & 190.46957588 & 197.33391953 & 199.38262717\end{array}$

The first five natural frequencies of the (a) built in-built in, (b) built in-pinned, and (c) pinned-pinned, uniform Trмosine nko beams have been determined using the technique of this paper. In each case with $n=20$, all frequencies were determined with less than $2.5 \%$ error and usually less than $1.0 \%$ error.

Example 2: We consider a truncated wedge built in at $x=0$ and made to slide at $x=L$. The width of the wedge is constant, but the height varies linearly along the length of the beam. The beam carries at $x=L$ a heavy mass, $M_{0}$, (see Fig. 2). In this problem, the end moment $\dot{S}_{L} \neq 0$,

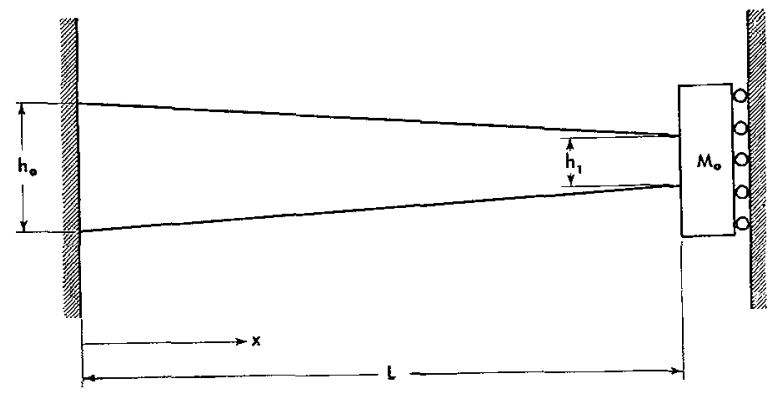

Fig. 2. Example 2

and we eliminate it through Castigliano's principle. Also $\dot{R}_{L} \neq 0$, but this is now the impulse on $M_{0}$ and hence becomes a variable in the final formulation of the problem.

We use the following parameters:

$$
\begin{aligned}
I_{0} & \ldots \text { Moment of inertia at } x=0 \\
A_{0} & \ldots \text { Cross sectional area at } x=0 \\
h_{0}, & h_{1} \ldots \text { Height of beam at } x=0, x=L \\
M_{s}, & M_{0} \ldots \text { Mass of the beam and the heavy block, } \\
& \ldots h_{1} / h_{0} \\
\beta & \ldots\left(1-h_{1} / h_{0}\right) \\
z_{i}, & \ldots x_{i} / L, x / L \\
r^{2} & \ldots I_{0} / A_{0} L^{2} \\
s^{2} & \ldots E I_{0} / k A_{0} G L^{2}
\end{aligned}
$$




$$
\begin{aligned}
a^{2} & \ldots\left(\eta / m L^{2}\right)=r^{2}\left(1+h_{1}^{2} / h_{0}^{2}\right) / 2, \\
\varepsilon & \ldots\left(n M_{0} / M_{s}\right)^{1 / 2} \\
r_{2 n+1} & \ldots(1 / \varepsilon) R_{L} .
\end{aligned}
$$

The kinetic energy, $T$, is

$$
\begin{gathered}
T=(1 / 2 m) \sum_{i=1}^{n} r_{i}{ }^{2}+(1 / 2 \eta) \sum_{\substack{i=1 \\
2 n+1}}^{n} \sigma_{i}{ }^{2}+\left(1 / 2 M_{0}\right) R_{L}{ }^{2}= \\
=(1 / 2 m) \sum_{i=1} r_{i}{ }^{2} .
\end{gathered}
$$

The bending moment and shear force distribution are given by

$$
\begin{gathered}
M(x)=\dot{S}_{L}+L\left[-\sum_{i=1}^{n} \dot{r}_{i}\left(z_{i}-z\right) U\left(z_{i}-z\right)+\right. \\
\left.+a \sum_{i=n+1}^{2 n} \dot{r}_{i} U\left(z_{i}-z\right)-\varepsilon \dot{r}_{2 n+1}(1-z)\right] \\
Q(x)=\sum_{i=1}^{n} \dot{r}_{i} U\left(z_{i}-z\right)+\varepsilon \dot{r}_{2 n+1} .
\end{gathered}
$$

The potential coenergy, $V^{*}$, becomes

$$
\begin{gathered}
V^{*}=\int_{0}^{1}\left(L / 2 E I_{0}\right)\left[M^{2} /(1-\beta z)^{3}+s^{2} L^{2} Q^{2} /(1-\beta z)\right] d z== \\
=V^{*}\left(\dot{r}_{1}, \dot{r}_{2}, \ldots, \dot{r}_{2 n+1}, \dot{S}_{L}\right) .
\end{gathered}
$$

The reaction $\dot{S_{L}}$ is eliminated by noting that the slope at the position $x=L$ is required to be zero. Thus by Castigliano's theorem

$$
\frac{\partial V^{*}}{\partial \dot{S}_{L}}=0 \text {. }
$$

Putting the expression for $\dot{S}_{L}$ from (d) into (c), and writing

$$
\begin{aligned}
& A(\xi)=\int_{0}^{\xi}\left[1 /(1-\beta z)^{3}\right] d z, \\
& B(\xi)=\int_{0}^{\xi}\left[z /(1-\beta z)^{3}\right] d z, \\
& C(\xi)=\int_{0}^{\xi}\left[z^{2} /(1-\beta z)^{3}\right] d z,
\end{aligned}
$$


we obtain the matrix $[A]$ for the problem:

$$
\begin{gathered}
0<i \leqslant n \quad 0<j \leqslant n \\
A_{i j}=\frac{L^{3}}{E I_{0}}\left\{z_{i} z_{j} A\left(z_{k}\right)-\left(z_{i}+z_{j}\right) B\left(z_{k}\right)+C\left(z_{k}\right)-\right. \\
\left.-\frac{\left[z_{i} A\left(z_{i}\right)-B\left(z_{i}\right)\right]\left[z_{j} A\left(z_{j}\right)-B\left(z_{j}\right)\right]}{A(1)}-\frac{s^{2} L n\left(1-\beta z_{k}\right)}{\beta}\right\} . \\
n<i \leqslant 2 n \quad n<j \leqslant 2 n \\
A_{i j}=\frac{L^{3}}{E I_{0}} a^{2}\left[A\left(z_{k}\right)-\frac{A\left(z_{i}\right) A\left(z_{j}\right)}{A(1)}\right] . \\
i=2 n+1 \quad j=2 n+1, \\
A_{i j}=\frac{L^{3}}{E I_{0}} \varepsilon^{2}\left[C(1)-\frac{B(1)^{2}}{A(1)}-\frac{s^{2} L n \alpha}{\beta}\right] . \\
0<i \leqslant n \quad n<j \leqslant 2 n \\
A_{i j}=\frac{L^{3}}{E I_{0}} a\left\{\frac{\left[z_{i} A\left(z_{i}\right)-B\left(z_{i}\right)\right] A\left(z_{j}\right)}{A(1)}-z_{i} A\left(z_{k}\right)+B\left(z_{k}\right)\right\} . \\
0<i \leqslant n \quad j=2 n+1 . \\
A_{i j}=\frac{L^{3}}{E I_{0}} \varepsilon\left\{\frac{\left[z_{i} A\left(z_{i}\right)-B\left(z_{i}\right)\right] B(1)}{A(1)}-z_{i} B\left(z_{i}\right)+C\left(z_{i}\right)-\frac{s^{2} L n\left(1-\beta z_{i}\right)}{\beta}\right\} . \\
n<i \leqslant 2 n \quad j=2 n+1 . \\
A L_{i j}=\frac{L^{3}}{E I_{0}} a \varepsilon\left[B\left(z_{i}\right)-\frac{A\left(z_{i}\right) B(1)}{A(1)}\right],
\end{gathered}
$$

where

$$
z_{k}=\min \left(z_{i}, z_{j}\right)
$$

The first five natural frequencies computed are listed below:

$$
r=0.02, s=0.05, \quad M_{0} / M_{s}=5.0, \alpha=0.5,
$$

Mode

$\begin{array}{lrr} & n=10 & n=20 \\ 1 & 0.94111269 & 0.94113062 \\ 2 & 13.84640980 & 13.84593678 \\ 3 & 36.33810806 & 36.36336756 \\ 4 & 67.60753536 & 67.73790646 \\ 5 & 105.10070515 & 105.78695011\end{array}$

\section{Acknowledgement}

One of the authors (BHK) wishes to acknowledge the partial support of Grant No. UI - 00025-03 from the National Institute of Health. 


\title{
References
}

[1] KARNOPP, B. H. and J. C. FUNG: On the approximate determination of natural frequencies and modes of cantilever beams. Acta Mech., IX, 109 (1970).

[2] HOANG, T. C.: Eigenvalues and modifying quotients of vibrations of beams. Univ. of Wis. Eng'g. Exp. Station Report No. 25, 1964.

\author{
Professor B. H. Karnopp and B. Nagendra \\ Department of Engineering Mechanies \\ College of Engineering \\ University of Michigan \\ Ann Arbor, Mich. 48104, U.S.A.
}

